EPJ Web of Conferences 38, 13001 (2012)

DOI: $10.1051 /$ epjconf/20123813001

(C) Owned by the authors, published by EDP Sciences, 2012

\title{
Proton-induced composite particle emission in inclusive reactions in the range of 100 to $200 \mathrm{MeV}$
}

\author{
A.A. Cowley ${ }^{1,2, a}$ \\ 1 Department of Physics, Stellenbosch University, Private Bag X1, Matieland 7602, South Africa \\ 2 iThemba Laboratory for Accelerator Based Sciences, P O Box 722, Somerset West 7129, South Africa
}

\begin{abstract}
Nucleon-induced inclusive reactions at incident energies in the 100 to $200 \mathrm{MeV}$ range, in which light composite particles are emitted, are of special interest. For emission of $\alpha$-particles into the continuum, it appears that the yield is enhanced towards lower outgoing energies of the ejectile compared with the value predicted by a multistep model. We postulate that a simple reaction mechanism, such as sequential decay from inelastic excitation, should be investigated more carefully as a possible explanation of the observed phenomenon. The motivation for this is that the suggested mechanism is a process which is observed in several $(p, p \alpha)$ knockout studies.
\end{abstract}

\section{Introduction}

The development of the exciton model of Griffin [1] represented a tremendous breakthrough in our appreciation of the physics mechanisms at play in the nuclear reactions leading to particle emission into the continuum. Further major developments were quantum mechanical formulations, first by Feshbach, Kerman and Koonin (FKK) [2], followed by Tamura, Udagawa and Lenske (TUL) [3], as well as Nishoika, Weidenmüller and Yoshida (NWY) [4]. These ideas were tested extensively, and they proved to be extremely valuable [5] to interpret experimental data - especially nucleon-induced nucleon emission at incident energies above $100 \mathrm{MeV}$.

Koning and Akkermans [6] investigated the theoretical relationships between the FKK, TUL and NWY models, and followed this up with a numerical study of the predictions of the model [7]. Although quantum mechanical formulations are very attractive from a theoretical point of view, application in terms of an extended version of the exciton model [8] remains attractive.

The code of Koning et al. [8,9] to calculate cross section distributions for nucleon-induced reactions for a comprehensive range of target masses and a wide range of incident energies, proves to be invaluable. Based on the two-component exciton model for nucleon emission, supplemented by an implementation of the principle of Kalbach systematics [10] for composite-particle emission, a remarkably successful reproduction of an extensive set of experimental distributions is achieved [8]. Therefore, with full justification it could be claimed that our understanding of pre-equilibrium reaction has reached a remarkably high degree of maturity. However, as I have pointed out recently [11], a number of inconsistencies are encountered in

\footnotetext{
a e-mail: aac@sun.ac.za
}

our attempts to describe especially inclusive $(p, \alpha x)$ reactions. Although many reasonably valid theoretical attempts to describe the experimental data share a basic intranuclear multistep interpretation, disconcerting differences in the specific reaction mechanism, that eventually lead to composite particle emission, emerges from a multitude of studies.

In this paper I will expand on those ideas presented at a recent international scientific meeting [11]. The basic contention is that perhaps sequential decay, after inelastic excitation, should be explored in order to explain the yield of $\alpha$-particles in proton-induced pre-equilibrium reactions. Although experimental evidence is somewhat sparse at present, this presentation could serve as an incentive for further investigation.

\section{Some general features of pre-equilibrium distributions}

A known feature of nucleon-induced pre-equilibrium cross section distributions, which complicates theoretical interpretation, is that in the region of interest to the present paper, they change rather slowly as a function of incident energy and target mass. Therefore, with a complicated reaction mechanism which embraces many reaction components, in combination with poor information of intermediate reaction products that lead to the observed particle emission, an understanding of the complete reaction mechanism is difficult. As mentioned by Koning et al. [8], their two-component exciton treatment contains many ingredients which can not be verified independently. Unfortunately the result is that a deficiency in one part of the model could be compensated with an unrelated aspect of the theory.

This is an Open Access article distributed under the terms of the Creative Commons Attribution License 2.0, which permits unrestricted use, distribution, and reproduction in any medium, provided the original work is properly cited. 


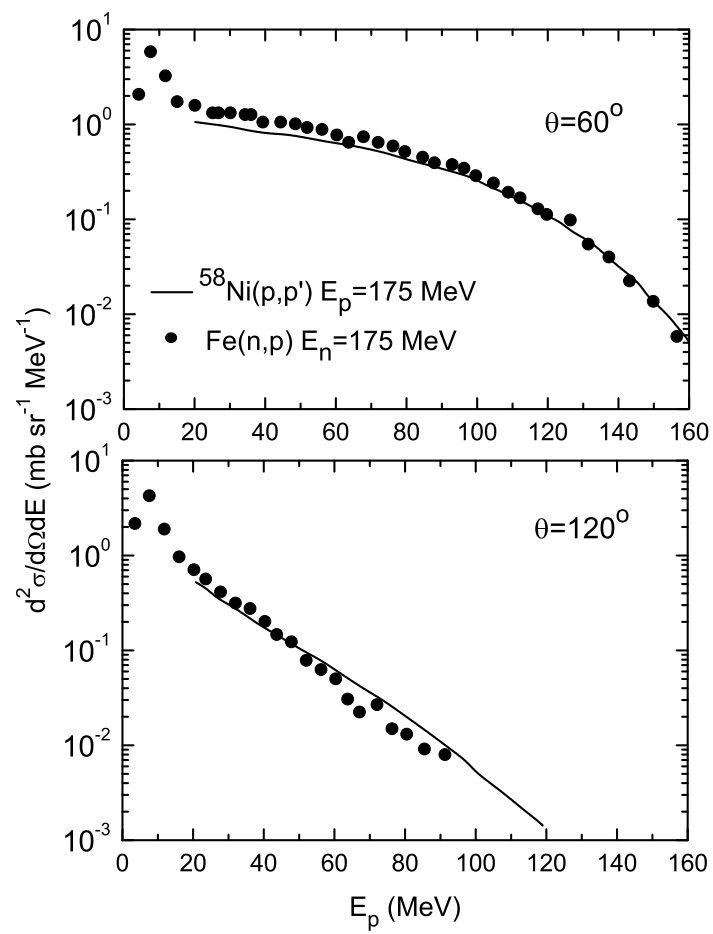

Fig. 1. Double differential cross section distribution for the reaction ${ }^{58} \mathrm{Ni}(p, p x)[12]$ is compared with results for the reaction ${ }^{n a t} \mathrm{Fe}(n, p x)$ [13] at an incident energy of $175 \mathrm{MeV}$ for both reactions. The data of Ref. [12] is shown as a curve drawn through those experimental points. For clarity of presentation, error bars, which are small anyway, are not shown. Data at the two indicated scattering angles are displayed.

A very interesting property of particle emission in nucleon-induced pre-equilibrium reactions is that experimental results appear to scale with incident energy and target species in a relatively simple way, which is much less sensitive to details of the nuclear system than theory would suggest. This property could perhaps provide guidance to the correct theoretical interpretation of the reaction mechanism.

For example, in figure 1 an experimental emission energy distribution for the reaction ${ }^{58} \mathrm{Ni}(p, p x)$ [12] is compared with results for the reaction ${ }^{n a t} \mathrm{Fe}(n, p x)$ at the same incident energy of $175 \mathrm{MeV}$ [13]. Data at two scattering angles, which are the same for both reactions are shown. The correspondence between these two data sets is remarkable, especially if we keep in mind that measurements with neutrons as projectiles present severe experimental challenges which could compromise the quality of the data [14] unless extreme care is taken. The similarity between the two sets clearly indicates that protons and neutrons react in the same way to a target nucleus, with the specific nuclear species not making a noticeable difference.

Another comparison, this time of the two reactions ${ }^{59} \mathrm{Co}\left(p,{ }^{3} \mathrm{He} x\right)[15]$ and ${ }^{n a t} \mathrm{Fe}\left(n,{ }^{3} \mathrm{He} x\right)[13]$ are shown in figure 2 . However, in this case not only are the targets and projectiles different, but the incident energy is $160 \mathrm{MeV}$ for the proton-induced reaction and $175 \mathrm{MeV}$ for the neutron projectile. By plotting results as a ratio of emission to incident energy, and so introduce a simple correction for the different incident energies at which the two reactions were measured, we again get excellent agreement between the two sets of data.

Lastly, in figure 3 , the reactions ${ }^{59} \mathrm{Co}(p, \alpha x)$ at an incident energy of $160 \mathrm{MeV}$ [17] and ${ }^{58} \mathrm{Ni}(p, \alpha x)$ [16] at 175 $\mathrm{MeV}$ are compared. Again there is a need to plot the cross sections as a function of the ratio of emission energy to incident energy.

Due to the widespread observed insensitivity to some details of the specific inclusive reaction, of which the quoted cases are just some examples, we assume in this paper that an effect observed for one, or a only a few target nuclei is representative of a far wider range. Incident energy dependence, relative to emission energy variation, is assumed to scale as a simple ratio as found in the examples.

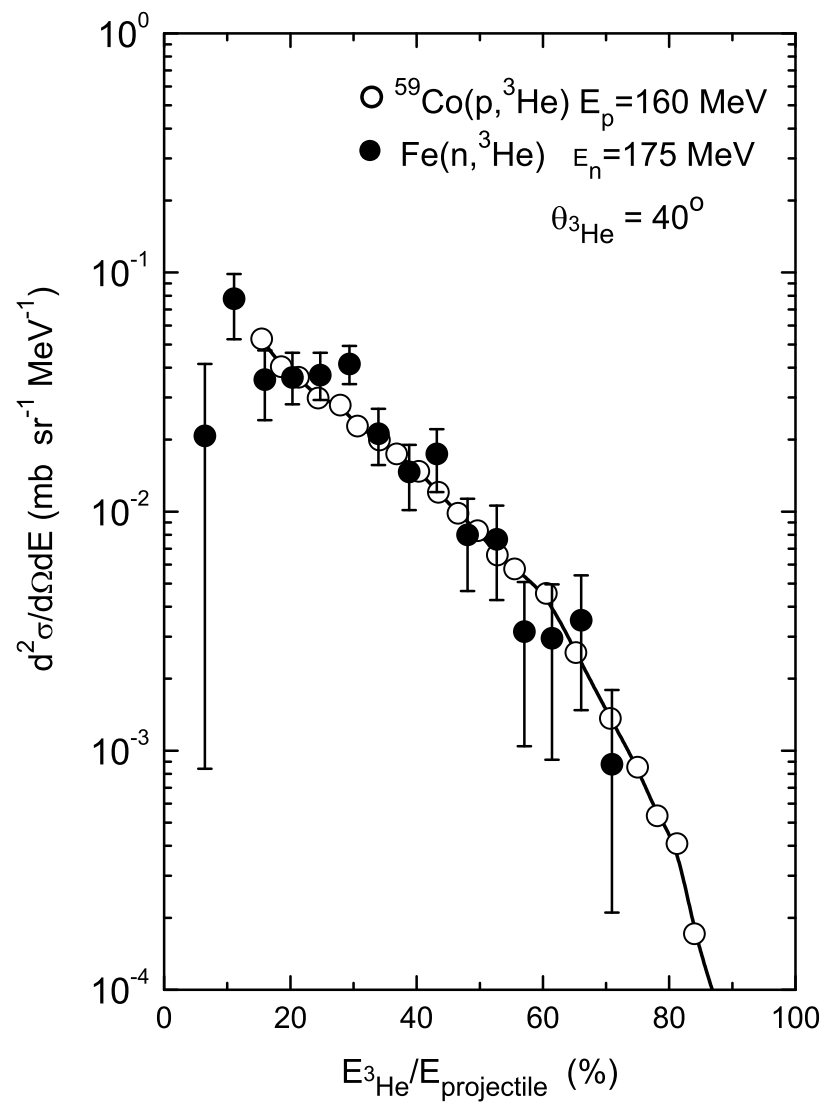

Fig. 2. Double differential cross section distribution for the reaction ${ }^{n a t} \mathrm{Fe}\left(n,{ }^{3} \mathrm{He} x\right)$ from Bevilacqua [13] at an incident energy of $175 \mathrm{MeV}$ compared with the results of Cowley et al. [15] for ${ }^{59} \mathrm{Co}\left(p,{ }^{3} \mathrm{He} x\right)$ at an incident energy of $160 \mathrm{MeV}$. To correct for the difference in incident energy, results are displayed as a function of the ratio of emitted ${ }^{3} \mathrm{He}$ energy to the incident energy of the projectile. 


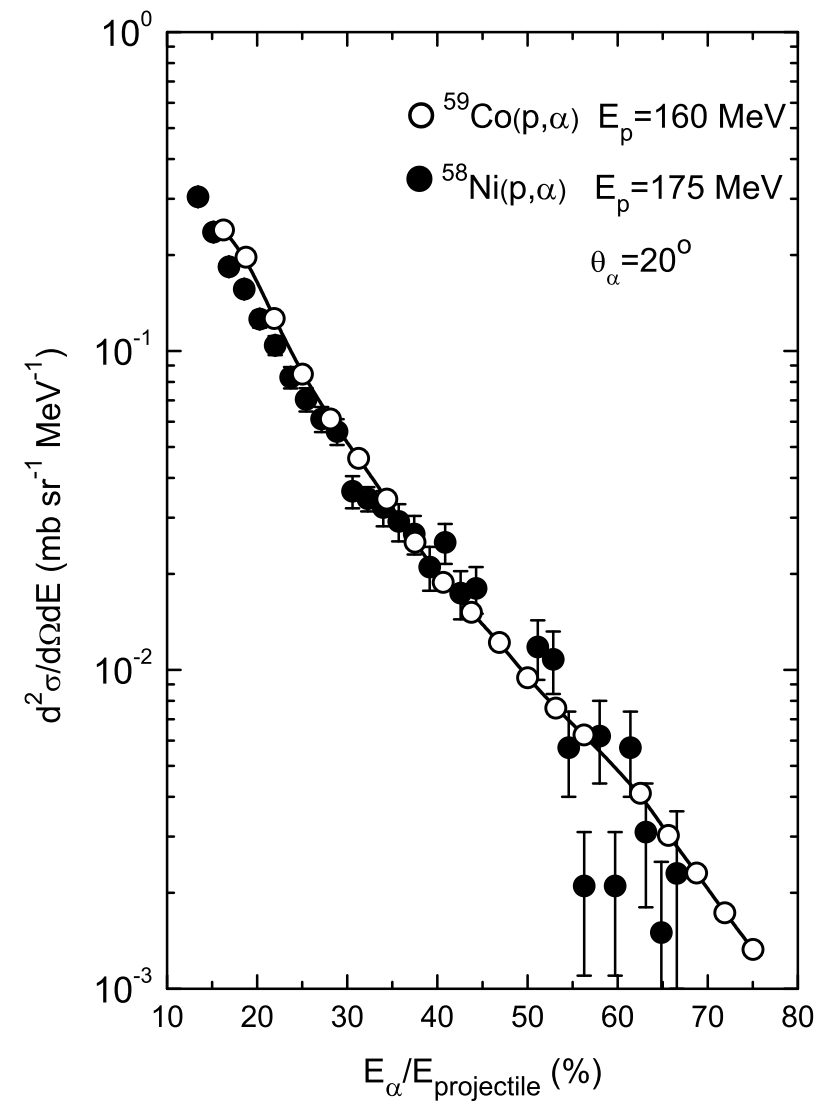

Fig. 3. Double differential cross section distribution for the reaction ${ }^{59} \mathrm{Co}(p, \alpha x)$ from Cowley et al. [15] an incident energy of 160 $\mathrm{MeV}$ compared with the results of Budzanowski et al. [16] for ${ }^{58} \mathrm{Ni}(p, \alpha x)$ at an incident energy of $175 \mathrm{MeV}$. For clarity of display, error bars are not shown for the ${ }^{59} \mathrm{Co}(p, \alpha x)$ reaction (those are smaller or comparable to the symbols anyway). To correct for the difference in incident energy, results are displayed as a function of the ratio of emitted $\alpha$-particle energy to the incident energy of the projectile.

\section{Theoretical treatment of proton-induced $\alpha$-particle emission}

In figure 4 the experimental ${ }^{59} \operatorname{Co}(p, \alpha x)$ double differential cross section emission energy distribution at an incident energy of $160 \mathrm{MeV}$ and a scattering angle of 20 degrees [17] is compared with theoretical predictions by Cowley et al. [17] and Bevilaqua [13]. In both predictions the $N N$ multistep part of the reaction mechanism was treated in a roughly equivalent way, although Ref. [13] used the programme TALYS [9] with its exciton-based formulation, and Ref. [17] employed a calculation traceable to FKK analyses of $(p, p x)$ studies in the appropriate incident energy and target mass range.

A major conceptual difference in the two theoretical analyses is the treatment of the final step of the compositeparticle production. The TALYS analysis suggests that, from the available options in the code, a purely pickup process should be selected for the final production of the ejectile. On the other hand, the calculation of Cowley et al. assumes a knockout reaction mechanism. Irrespective of this crucial difference in mechanism, both predictions clearly fail at an $\alpha$-particle emission energy lower that about 100 $\mathrm{MeV}$. Furthermore, a combination of pickup and knockout would not address the deficiency.

Although the underestimation of the yield towards lower $\alpha$-particle emission energies is displayed at only one scattering angle in figure 4 , the phenomenon appears to apply to all scattering angle. This is shown in figure 5 at two emission energies for the same reaction as in figure 4 , namely ${ }^{59} \mathrm{Co}(p, \alpha x)$ at an incident energy of $160 \mathrm{MeV}$. Whereas good agreement between theoretical prediction and experimental yield is found at an emission energy of $120 \mathrm{MeV}$, a difference of an order of magnitude is observed over the whole angular range at an outgoing energy of 70 $\mathrm{MeV}$. It should be mentioned that in the analysis of Cowley et al. [17] the theory is normalized at high emission energies, but based on sound theoretical arguments related to details of the calculations, this should be a correct procedure.

The interesting feature of figure 4 is that two contradictory analysis of the same data set display the same qualitative underestimation at low $\alpha$-particle emission energies.

\section{Possible reaction mechanisms to explain the low yield of emitted $\alpha$-particles}

We will assume that the phenomenon that we discussed in the previous section is not due to an inadequacy of the theoretical formulations, or their implementation, but rather a missing part of the reaction mechanism that drives protoninduced $\alpha$-particle emission in pre-equilibrium emission. However, note that some theoretical approaches do not seem to suffer from the same problem (see e.g. Ref. [18]).

Another interpretation is that, in addition to the normal multistep component, the additional yield in $\alpha$-particle emission is greatly determined by a fragmentation mechanism of the target [16]. In this model, during the cascade phase from which emission also occurs, the excess of $\alpha$ particles mainly comes from a fast-moving source from breakup of the target, which co-exists with a slow-moving source and a fireball. Although the ideas appear to be consistent with studies $[19,20]$ at incident energies in the $\mathrm{GeV}$ range, it is difficult to see how these mechanisms could extrapolate seamlessly down to about $100 \mathrm{MeV}$. Furthermore, these alternative and supplementary treatments have not been tested to the same extent as, for example, predictions of TALYS [9].

A simple inelastic mechanism for the enhanced $\alpha$ particle yield in proton-induced inclusive reactions is proposed. We suggest that one should explore a source of $\alpha$ particle emission that is observed prominently in exclusive $(p, p \alpha)$ knockout studies [21-24]. In those studies it is observed that low-lying states in the target system, which undergo sequential $\alpha$-particle decay, are inelastically excited. 


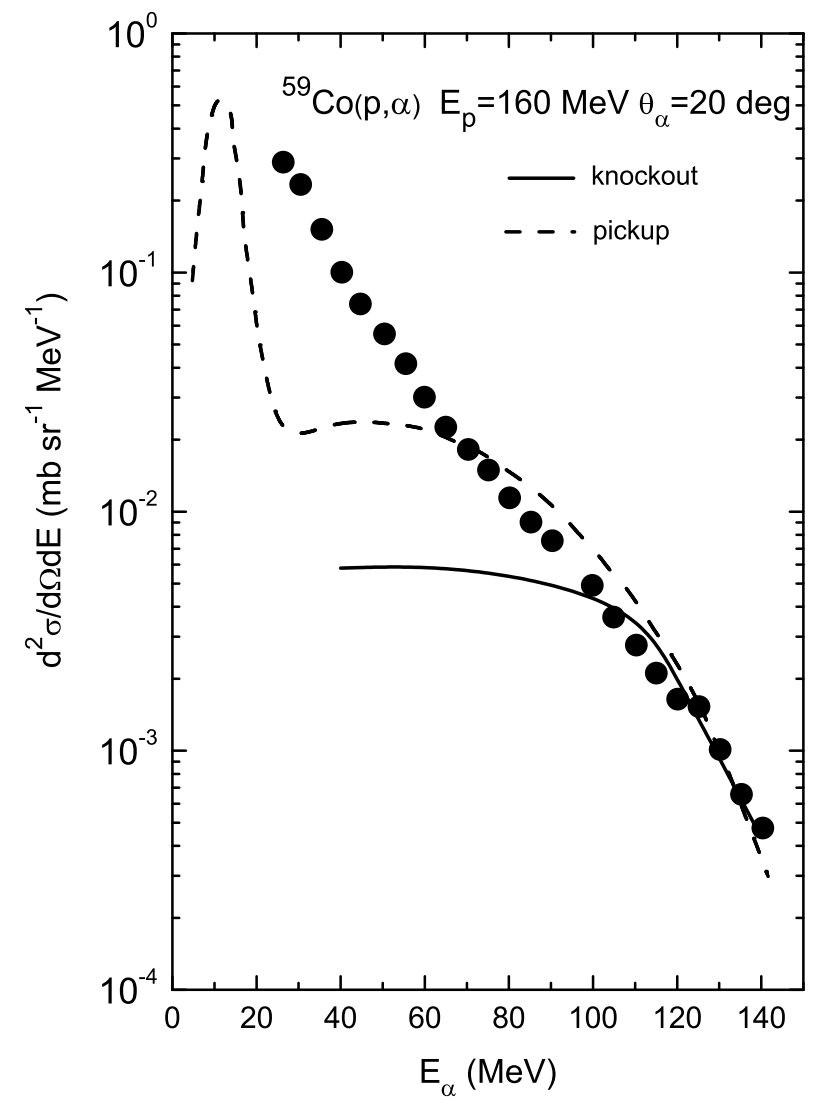

Fig. 4. Double differential cross section distribution for the ${ }^{59} \mathrm{Co}(p, \alpha x)$ reaction at an incident energy of $160 \mathrm{MeV}$. The experimental data of Cowley et al. [17] are shown with error bars which are smaller than the symbol size. The curves represent preequilibrium calculations by Bevilacqua [13] for a pickup process as a final step (dashed line), and a prediction of Cowley et al. (solid line) which assumes a knockout mechanism instead.

Although explicit calculations are difficult at this stage, additional experiments that should enable quantitative cross section calculations to be performed, will be suggested. Note that the virtue of the proposed experiments and analysis does not depend on whether either a knockout or a nucleon transfer process drives the $\alpha$-particle emission at higher energies.

In the following section evidence, which serves as justification for the hypothesis, is presented.

\section{Distributions in exclusive $(p, p \alpha)$ reactions}

As was already mentioned, a reason for the observed discrepancy between theory and experimental data of inclusive ( $p, \alpha x)$ towards low $\alpha$-particle emission energy is suggested by features seen in several $(p, p \alpha)$ knockout studies. Knockout reactions are understood well and the DWIA re-

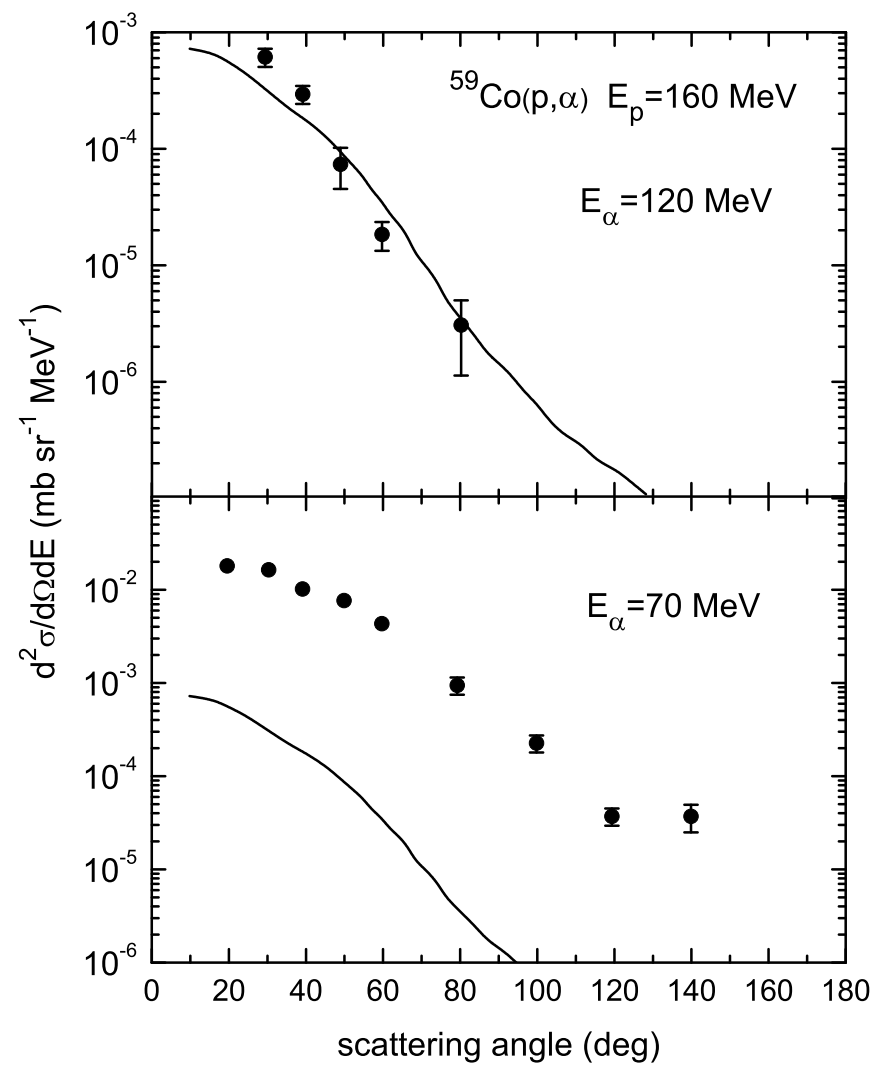

Fig. 5. Double differential cross section angular distributions for the ${ }^{59} \mathrm{Co}(p, \alpha x)$ reaction at an incident energy of $160 \mathrm{MeV}$ at two emission energies as indicated in the figure. The curves are preequilibrium calculations in which knockout in the final step is assumed. All results are adopted from the paper of Cowley et al. [17].

produces features of experimental distributions very well (see e.g. [21-24]). The kinematic geometry that is mostly investigated, is a quasifree arrangement in which the scattering angles are selected to be coplanar on opposite sides of the incident beam in such a way that zero recoil momentum of the heavy residual nucleus is allowed. A locus of events corresponding to a specific final state of the residual nucleus is selected, and the available kinetic energy is shared mainly between the two light outgoing particles. The cross section can be presented as a function of the energy of either the emerging proton, or $\alpha$-particle.

In $(p, p \alpha)$ reactions it is well known that sequential decay [21] of $\alpha$-particles as a result of inelastic excitations of the target nucleus interfere with the knockout component. The decay process becomes progressively conspicuous towards larger proton-emission angles, corresponding to smaller $\alpha$-particle scattering angles. Typical examples [22] of the cross section distributions for the reaction ${ }^{12} \mathrm{C}(p, p \alpha){ }^{8} \mathrm{Be}$ (g.s.) reaction, at an incident energy of $100 \mathrm{MeV}$, is shown in figure 6. The plot is shown a function of $\alpha$-particle kinetic energy, in other words the ( $p, \alpha p)$ 


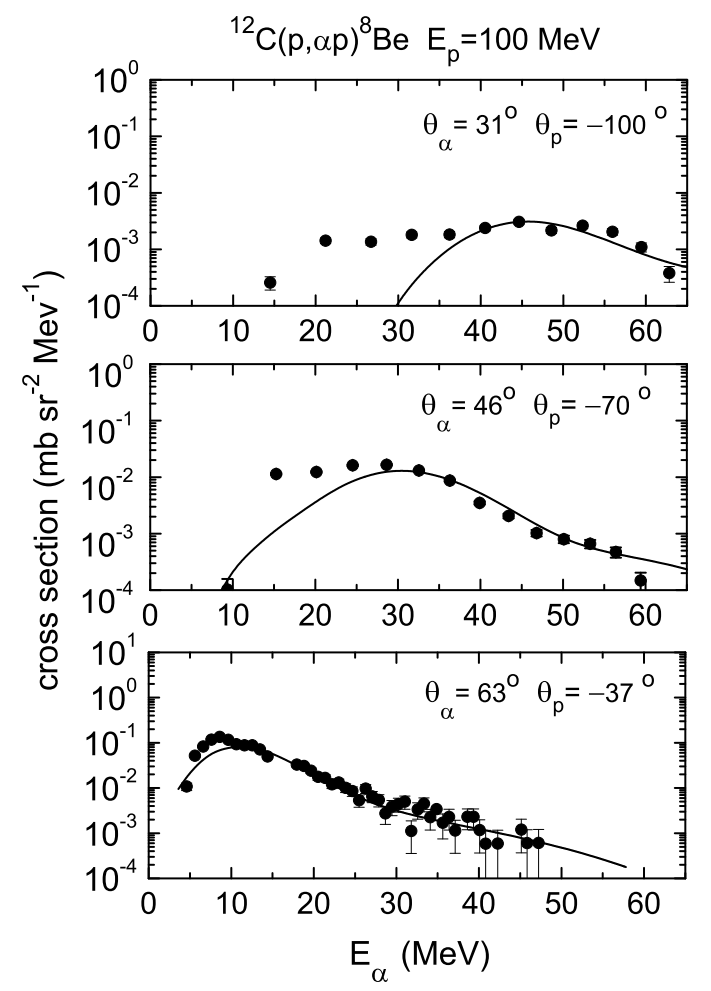

Fig. 6. Energy sharing cross section distributions extracted from the study of Mabiala et al. [22] for the ${ }^{12} \mathrm{C}(p, \alpha p){ }^{8} \mathrm{Be}$ knockout reaction at an incident energy of $100 \mathrm{MeV}$ at quasifree angle pairs as indicated in the figure. Results are shown as a function of $\alpha$ particle kinetic energy. The curves represent distorted-wave impulse approximation (DWIA) $[21,25,26]$ predictions for a pure knockout reaction mechanism [22].

knockout data in figure 6 were derived from the $(p, p \alpha)$ results of Mabiala et al. [22]. Results are shown at $\alpha$-particle scattering angles as listed in the figure.

Figure 6 clearly displays yield in excess of the knockout contributions, which are shown as curves. Based on a number of independent criteria it is safe to assume that the DWIA correctly estimates the contribution of direct knockout [22] at each scattering angle pair.

Note that the contribution to the cross section in excess of the knockout yield becomes more prominent towards more forward scattering angles of the $\alpha$. The yield also extends to higher $\alpha$-particle kinetic energies as this scattering angle decreases.

It appears that the phenomenon observed for ${ }^{12} \mathrm{C}$ applies also to a heavier nucleus such as ${ }^{40} \mathrm{Ca}$ [23] at an incident energy of $100 \mathrm{MeV}$. It is also present at higher projectile energy [24], which is much beyond the $200 \mathrm{MeV}$ or so of interest to this investigation.

In inclusive ( $p, \alpha x)$ reactions knockout of $\alpha$-clusters, as seen in explicit coincidence experiment, should contribute. In addition, the sequential decay mechanism should be even more prominent than figure 6 suggests. The reason is that, due to the very small solid angle available to knockout kinematics compared to inelastic excitation, the rel- ative contribution of knockout to the continuum yield is undoubtedly much lower than for sequential decay. One should keep in mind that the results shown in figure 6 were measured at angle pairs where knockout cross sections are at a maximum.

\section{Summary and conclusion}

It is known that in nucleon-induced reactions, in the incident energy range of 100 to $200 \mathrm{MeV}$, the intranuclear nucleon-nucleon cascade is a fundamental mechanism that drives the pre-equilibrium process. In theoretical predictions of inclusive reactions, the yield of composite ejectiles such as $\alpha$-particles, generally appears to be underestimated when the emission energy drops to roughly half of the kinematically-allowed maximum. This phenomenon becomes increasingly more noticeable as the ejectile energy drops towards values approaching emission from the compound nucleus.

There are, of course, many alternative theoretical approaches to address the problem. For example, the observed deficiency may be resolved by postulating fragmentation of the target nucleus followed by emission from a fast moving source. However, such a process is normally associated with reactions at much higher incident nucleon energies, in the $\mathrm{GeV}$ range. Consequently it is not clear how such mechanism could extrapolate towards lower incident energies around 100 to $200 \mathrm{MeV}$.

A simple process, which is in principle also able to account for the observed effect, is the participation of sequential $\alpha$-particle decay from inelastically excited states in the target nucleus. This type of reaction mechanism is very often encountered in $(p, p \alpha)$ investigations in the incident energy range currently of interest, where it merely serves to interfere with the knockout reaction of primary concern.

Consequently, sequential $\alpha$-particle decay from lowlying inelastic excitations seems to be a serious contender as a source of yield in inclusive $(p, \alpha)$ reactions at low emission energies. Experiments need to be performed on the $(p, p \alpha)$ reaction for various target nuclei and for a range of kinematic conditions which are very far from the region of interest to knockout.

\section{Acknowledgement}

This work was performed with funding from the South African National Research Foundation (NRF). The financial support is gratefully acknowledged.

\section{References}

1. J.J. Griffin, Phys. Rev. Lett. 17, 478 (1966)

2. H. Feshbach, A. Kerman and S. Koonin, Ann. Phys. (NY) 125, 429 (1980)

3. T. Tamura, T. Udagawa, and H. Lenske, Phys. Rev. C 26, 379 (1982) 
4. H. Nishoika, H.A. Weidenmüller, and H. Yoshida, Ann. Phys. (NY) 183, 166 (1988)

5. E. Gadioli and P.E. Hodgson, Pre-equilibrium Nuclear Reactions (Oxford University Press, New York, 1991)

6. A.J. Koning and J.M. Akkermans, Ann. Phys. (NY) 208, 216 (1991)

7. A.J. Koning and J.M. Akkermans, Phys. Rev. C 47, 724 (1991)

8. A.J. Koning and M.C. Duijvestijn, Nucl. Phys. A 744, 15 (2004)

9. A.J. Koning, S. Hilaire, and M.C. Duijvestijn, in: Proceedings of the Intl. Conference on Nuclear Data for Science and Technology (Nice, France, April 22-27, 2007), eds. O. Bersillon, F. Gunsing, E. Bauge, R. Jacqmin, and S. Leray, EDP Sciences, 2008, p. 211.

10. C. Kalbach, Phys. Rev. C 37, 2350 (1988)

11. A.A. Cowley, in: Proceedings of the Third Intl. Workshop on Compound-Nuclear Reactions and Related Topics (CNR*11), (Prague, Czech Republic, September 1923, 2011), eds. M. Krtička, F. Bečvář, and J. Kroll, EPJ Web of Conf. 21, 09002 (2012)

12. S.V. Förtsch, A.A. Cowley, J.J. Lawrie, D.M. Whittal, J.V. Pilcher, and F.D. Smit, Phys. Rev. C 43, 691 (1991)

13. R. Bevilacqua, PhD thesis, Uppsala University (2011)

14. R. Bevilacqua, S. Pomp, V. Simutkin, M. Hayashi, S. Hirayama, Y. Naitou, Y. Watanabe, U. Tippawan, M. Tesinsky, G. Ban, J.-L. Lecouey, N. Marie, and Q. Hamel, Nucl. Instr. Meth. Phys. A 646, 100 (2011)

15. A.A. Cowley, G.J. Arendse, G.F. Steyn, J.A. Stander, W.A. Richter, S.S. Dimitrova, P. Demetriou, and P.E. Hodgson, Phys. Rev. C 55, 1843 (1997)

16. A. Budzanowski, M. Fidelus, D. Filges, F. Goldenbaum, H. Hodde, L. Jarczyk, B. Kamys, M. Kistryn, St. Kistryn, St. Kliczewski, A. Kowalczyk, E. Kozik, P. Kulessa, H. Machner, A. Magiera, B. Piskor-Ignatowicz, K. Pysz, Z. Rudy, R. Siudak, and M. Wojciechowski, Phys. Rev. C 80, 054604 (2009)

17. A.A. Cowley, G.J. Arendse, J.W. Koen, W.A. Richter, J.A. Stander, G.F. Steyn, P. Demetriou, P.E. Hodgson, and Y. Watanabe, Phys. Rev. C 54, 778 (1996)

18. S. Kunieda, T. Kawano, M.B. Chadwick, T. Fukahori, and Y. Watanabe, in: Proceedings of the Third Intl. Workshop on Compound-Nuclear Reactions and Related Topics (CNR*11), (Prague, Czech Republic, September 1923, 2011), eds. M. Krtička, F. Bečvář, and J. Kroll, EPJ Web of Conf. 21, 09003 (2012)

19. A. Budzanowski, M. Fidelus, D. Filges, F. Goldenbaum, H. Hodde, L. Jarczyk, B. Kamys, M. Kistryn, St. Kistryn, St. Kliczewski, A. Kowalczyk, E. Kozik, P. Kulessa, H. Machner, A. Magiera, B. Piskor-Ignatowicz, K. Pysz, Z. Rudy, R. Siudak, and M. Wojciechowski, Phys. Rev. C 78, 024603 (2008)

20. A. Budzanowski, M. Fidelus, D. Filges, F. Goldenbaum, H. Hodde, L. Jarczyk, B. Kamys, M. Kistryn, St. Kistryn, St. Kliczewski, A. Kowalczyk, E. Kozik, P. Kulessa, H. Machner, A. Magiera, B. Piskor-Ignatowicz, K. Pysz, Z. Rudy, R. Siudak, and M. Wojciechowski, Phys. Rev. C 82, 034605 (2010)

21. P.G. Roos, N.S. Chant, A.A. Cowley, D.A. Goldberg, H.D. Holmgren, and R. Woody, Phys. Rev. C 15, 69
(1977)

22. J. Mabiala, A.A. Cowley, S.V. Förtsch, E.Z. Buthelezi, R. Neveling, F.D. Smit, G.F. Steyn, and J.J. van Zyl, Phys. Rev. C 79, 054612 (2009)

23. T.A. Carey, P.G. Roos, N.S. Chant, A. Nadasen, and H.L. Chen, Phys. Rev. C 29, 1273 (1984)

24. T. Yoshimura, A. Okihana, R.E.Warner, N.S. Chant, P.G. Roos, C. Samanta, S. Kakigi, N. Koori, M. Fujiwara, N. Matsuoka, K. Tamura, E. Kubo, and K. Ushiro, Nucl. Phys. A 641, 3 (1998)

25. N.S. Chant and P.G. Roos, Phys. Rev. C 15, 57 (1977)

26. N.S. Chant and P.G. Roos, Phys. Rev. C 27, 1060 (1983) 